\title{
The effect of baicalin in a mouse model of retinopathy of prematurity
}

\author{
Hyoung Jo, ${ }^{1, \#}$, Sang Hoon Jung ${ }^{1, \#}$, Hye Bin Yim ${ }^{2}$, Sung Jin Lee ${ }^{3}$ E Kui Dong Kang ${ }^{2, *}$ \\ ${ }^{1}$ Functional Food Center, Korea Institute of Science and Technology (KIST) Gangneung Institute, Gangneung 210-340, ${ }^{2}$ Department of \\ Ophthalmology, Incheon St. Mary's Hospital, College of Medicine, The Catholic University of Korea, Incheon 403-720, ${ }^{3}$ Department of \\ Ophthalmology, College of Medicine, Soonchunhyang University, Seoul 140-743, Korea
}

\begin{abstract}
Baicalin is a flavonoid derived from the dried root of Scutellaria baicalensis. In this study, oxygen-induced retinopathy was used to characterize the anti-angiogenic properties of baicalin in mice. Pups were exposed to a hyperbaric oxygen environment to induce retinal angiogenesis and were subjected to intraperitoneal injection of baicalin. Avascular area, neovascular tufts, and neovascular lumens were quantified from digital images. Compared to the vehicle, baicalin clearly reduced the central avascular zone and the number of neovascular tufts and lumens. High-dose baicalin $(10 \mathrm{mg} / \mathrm{kg})$ significantly reduced the expression of matrix metalloproteinase-2 (MMP-2), MMP-9, angiotensin II, and vascular endothelial growth factor (VEGF). These results show that baicalin is a powerful antiangiogenic compound that attenuates new vessel formation in the retina after systemic administration, and is a candidate substance for therapeutic inhibition of retinal angiogenesis. [BMB Reports 2015; 48(5): 271-276]
\end{abstract}

\section{INTRODUCTION}

Retinopathy of prematurity (ROP) is a disease of the eye that affects premature babies, in which exposure to high levels of oxygen during a particular stage of retinal vascular development results in retinal neovascularization and retinal detachment. Although the incidence of this sight-threatening disease has been dramatically decreased over recent decades, it is still a major cause of blindness in children (1). ROP is recognized by a typical elevated ridge and characteristic extra-retinal neovascularization, and current medical or surgical strategies are directed at preventing abnormal formation of new vessels.

${ }^{*}$ Corresponding author. Tel: +82-32-280-5110; Fax: +82-32-2805114; E-mail: kuidongkang@gmail.com

${ }^{\text {\#}}$ Shared first authorship since they contributed equally.

http://dx.doi.org/10.5483/BMBRep.2015.48.5.131

Received 17 June 2014, Revised 11 July 2014, Accepted 16 August 2014

Keywords: Angiogenesis, Angiotensin II, Baicalin, Oxygen-induced retinopathy, VEGF
Evidence from randomized multicenter trials supports this treatment approach by showing that laser photocoagulation and/or cryotherapy reduces the progression of ROP and prevents more debilitating complications such as retinal detachment or macular dragging $(2,3)$. In addition, intravitreal injections of anti-VEGF agents are now a new trend in the treatment of ROP and have been shown to be relatively effective in inhibiting pathologic retinal angiogenesis. However, these treatment modalities have high rates of systemic complications, which have led to new approaches using anti-angiogenic agents $(4,5)$. One such approach is to discover natural bioactive products from plants that are expected to have low systemic toxicity. Efforts to discover natural compounds with powerful anti-angiogenic activity are of significant medical value, as these orally ingestible compounds are free from the complications associated with invasive surgical procedures.

Baicalin (7-D-glucuronic acid,5,6-dihydroxyflavone) is a flavonoid derived from the dried root of Scutellaria baicalensis. Several studies have investigated the anti-angiogenic activity of baicalin $(6,7)$; however, studies evaluating baicalin in ocular tissue or retinal angiogenesis are scarce. Thus, investigating the potential of baicalin to attenuate new vessel formation in a model of oxygen-induced retinopathy is worthy of study, as such data may lead to alternative treatments for patients with various ocular diseases where abnormal angiogenesis is the main pathophysiology. Interestingly, Lui et al. (6) demonstrated that baicalin inhibits angiogenesis by inhibiting basic fibroblast growth factor (bFGF) and matrix metalloproteinase-2 (MMP-2), whereas Zhang et al. showed that baicalin promotes angiogenesis by inducing vascular endothelial growth factor (VEGF) expression through the activation of the estrogen-related receptor (ERR $\alpha$ ) pathway (8). Therefore, two distinct and seemingly contradictory properties of baicalin have been described, and thus the effect of baicalin on new vessel formation remains a matter of debate, and it is vital to gain further information on this substance. In this report, we show for the first time that intraperitoneal administration of baicalin inhibits retinal neovascularization in a mouse model of oxygen-induced retinopathy (OIR). 


\section{RESULTS}

Baicalin down-regulates the expression of MMP-2, MMP-9, angiotensin II, and VEGF

The levels of pro-angiogenic proteins such as MMP-2, MMP-9, angiotensin II, VEGF, and CD31 in the retina relative to GAPDH are shown in Fig. 1. These data show that exposure to hyperbaric conditions from $\mathrm{P} 12$ to $\mathrm{P} 17$ clearly increased retinal protein levels of MMP-2, MMP-9, VEGF, and CD31. Baicalin at a high dose $(10 \mathrm{mg} / \mathrm{kg})$ significantly reduced the expression of these pro-angiogenic proteins, whereas this effect was not observed in the low-dose $(1 \mathrm{mg} / \mathrm{kg})$ group. Baicalin did not affect CD31 abundance at any tested dose. In the retinas of pups exposed to hyperbaric conditions from P12 to P17, angiotensin II levels were significantly increased, and highdose baicalin $(10 \mathrm{mg} / \mathrm{kg})$ significantly inhibited this up-regulation $(\mathrm{P}<0.05$, Fig. $1 \mathrm{~B})$. There were no significant differences in the expression of any evaluated protein between the vehicle and low-dose baicalin groups (Mann-Whitney $U$ test).

\section{Baicalin significantly reduces the central non-perfusion area and retinal tufts in flat mounts}

To quantify the central non-perfusion area and retinal tufts, retinal flat mounting using FITC-dextran was performed. The retinas of pups exposed to hyperbaric conditions from P12 to P17 clearly showed a central avascular area (Fig. 2A). In the retinas of the sham-injected group, $38.26 \pm 4.41 \%$ of the total retinal area was avascular (Fig. 2B, Table 1), whereas the retinas of pups treated with low-dose (1 mg/kg, Fig. 2E) or high-dose (10 $\mathrm{mg} / \mathrm{kg}$, Fig. $2 \mathrm{H}$ ) baicalin showed avascular retinal areas of $21.45 \pm 3.14 \%$ and $19.34 \pm 3.68 \%$, respectively, which were significantly different from that of the sham-operated group $(P$ $<0.05)$. However, there were no significant differences between the avascular areas in pups treated with low-dose (1 $\mathrm{mg} / \mathrm{kg}$ ) and high-dose $(10 \mathrm{mg} / \mathrm{kg})$ baicalin. SWIFT_NV was used to quantify the retinal neovascular tufts on superficial vascular plexuses (Fig. 2C, 2F, and 2I). Neovascular tufts on superficial vascular plexuses were prominent in the sham-injected group, and made up $4.35 \pm 0.71 \%$ of the total retina. In pups treated with low-dose $(1 \mathrm{mg} / \mathrm{kg})$ or high-dose $(10$ $\mathrm{mg} / \mathrm{kg}$ ) baicalin, neovascular tufts were significantly decreased $(\mathrm{P}<0.05)$. However, there were no significant differences in retinal neovascular tufts between pups treated with low-dose $(1 \mathrm{mg} / \mathrm{kg})$ and high-dose $(10 \mathrm{mg} / \mathrm{kg})$ baicalin.

Baicalin significantly reduces the number of vascular lumen Sagittal retina sections were stained with hematoxylin and eo$\sin (\mathrm{H} \& \mathrm{E})$ and vascular lumens of new vessels growing into the vitreous were counted. In the vehicle-treated group (Fig. 3A), the average number of vascular lumens was $21.09 \pm 3.17$, whereas the low-dose $(1 \mathrm{mg} / \mathrm{kg}$, Fig. 3B) and high-dose (10 $\mathrm{mg} / \mathrm{kg}$, Fig. 3C) baicalin groups showed $18.63 \pm 2.37$ and $12.27 \pm 1.27$ vascular lumens, respectively. High-dose baicalin significantly reduced the number of vascular lumens in the retina after exposure to hyperbaric conditions (Fig. 3D, P < 0.05).
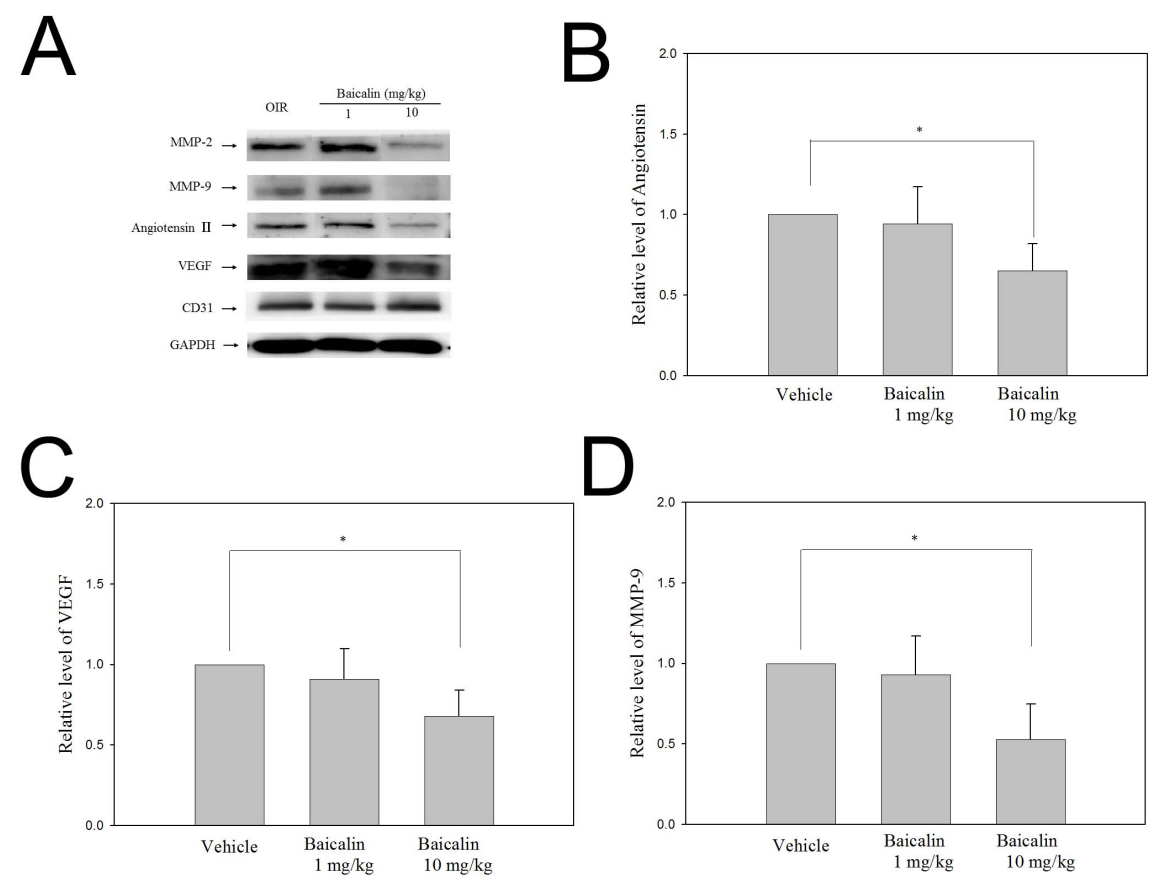

Fig. 1. Western blot analysis of the proteins expressed in the retina following oxygen-induced retinopathy. Exposure to hyperbaric condition increased the levels of matrix metalloproteinase-2 (MMP-2), MMP-9, angiotensin II, and vascular endothelial growth factor (VEGF), and high-dose baicalin (10 mg/ $\mathrm{kg})$ significantly reduced the expression of these proteins. The expression of CD31 was not affected by baicalin at any dose. Protein levels were normalized to GAPDH. 


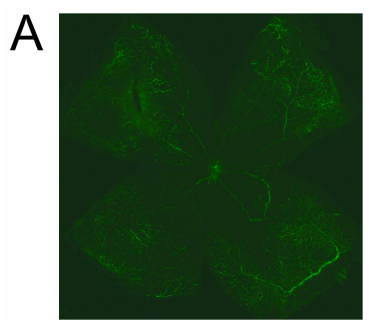

B

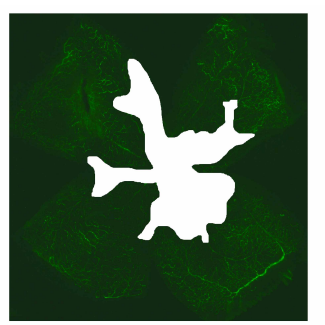

D
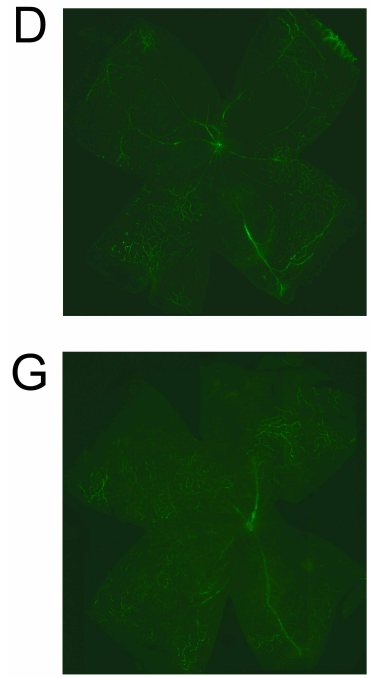

E

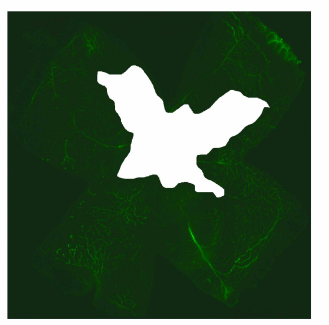

$\mathrm{H}$

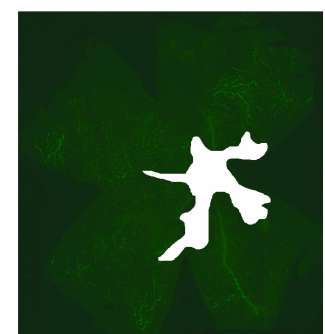

C

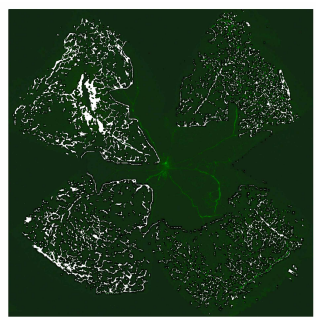

F

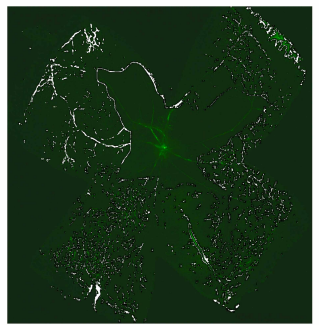

1

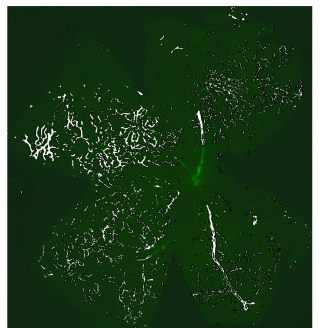

Fig. 2. FITC-dextran-perfused retinal flat mounts showing retinal vascularization (left column), avascular area (middle column indicated as white area), and neovascular tufts (right column). The central ischemic area of the vehicleinjected group (B) was larger than that of the baicalin-treated groups $(E, H)$. In the vehicle-injected group, many neovascular tufts were observed (C), but their occurrence was reduced by low-dose (1 mg/kg) (F) and high-dose (10 mg/kg) (I) baicalin.

Table 1. Quantification of avascular area and neovascular tufts

\begin{tabular}{lrrr}
\hline & Vehicle & Baicalin 1 mg/kg & Baicalin 10 mg/kg \\
\hline Avascular area (percentage of total retinal area) & $38.26 \pm 4.41$ & $21.45 \pm 3.14$ & $19.34 \pm 3.68$ \\
Neovascular tufts (percentage of total retinal area) & $4.35 \pm 0.71$ & $2.89 \pm 0.34$ & $3.02 \pm 0.41$ \\
\hline
\end{tabular}

*Statistical significance was tested by one-way ANOVA followed by Kruskal-Wallis test. Data expressed as mean \pm standard deviation.

\section{DISCUSSION}

The major purpose of this study was to deduce whether in vivo evidence could be generated to show that baicalin inhibits retinal neovascularization and to investigate the underlying mechanisms by which baicalin inhibits new vessel formation in the retina. As stated in the introduction, conflicting results using various in vivo and in vitro models were observed regarding the effect of baicalin in new vessel formation. To provide in vivo evidence with regard to this issue and define the role of baicalin in ocular tissue, the experiments conducted in this study used an oxygen-induced retinopathy model. Similar to our observations, a previous study used a laser-induced choroidal neovascular model to show that intravitreal injection of baicalin effectively attenuates choroidal neovascularization (9). Although intravitreal injection is one of the most effective ways to deliver baicalin to the tissue, this invasive procedure carries several risks and complications. Moreover, the eyes of the pups are relatively small and fragile, and conducting intravitreal injections in these animals is extremely challenging. Surprisingly, in our study, intraperitoneal baicalin administration attenuated new retinal vessel formation in a mouse model of OIR, and also significantly reduced the expression of pro-angiogenesis proteins MMP-2, MMP-9, angiotensin II, and VEGF.

In our study, baicalin effectively inhibited retinal expression of angiotensin II in a mouse model of OIR. This is the first report to show that baicalin can down-regulate angiotensin II in the retina, and the precise mechanism by which this occurs is a matter for speculation. However, it can be hypothesized that baicalin inhibits angiotensin by blocking the actions of renin and angiotensin-converting enzyme (ACE). This hypothesis is 

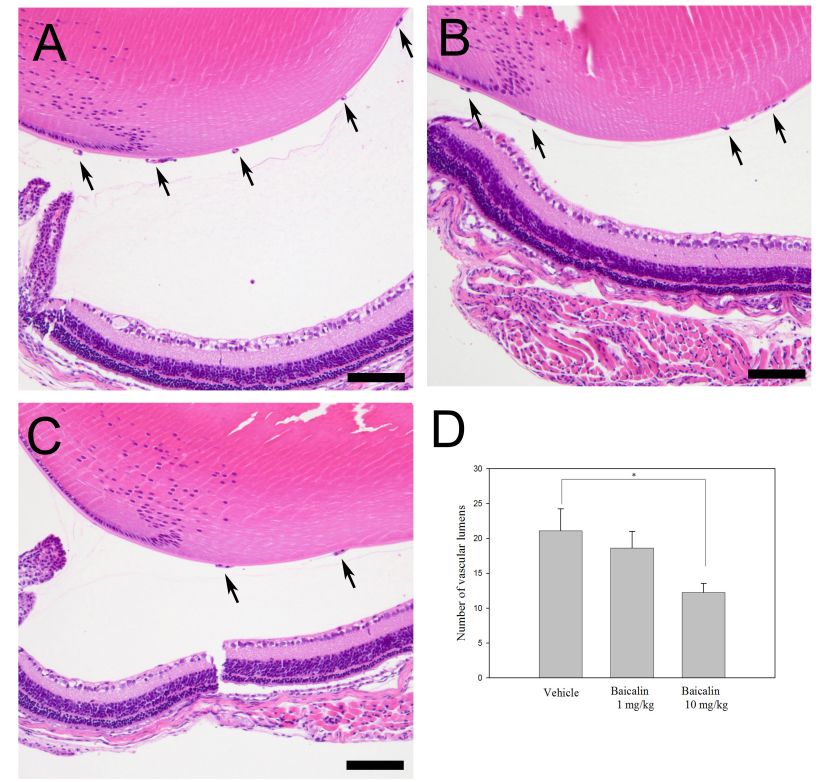

D

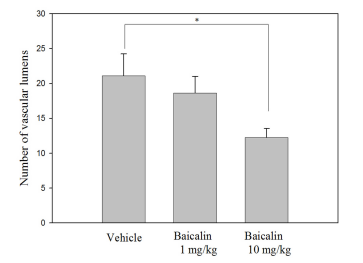

Fig. 3. Sectioning of the retina after hematoxylin and eosin staining to quantify neovascular lumens. Many neovascular lumens were observed in the vehicle-injected group (A), whereas this extensive neovascularization was significantly reduced in the low-dose (1 $\mathrm{mg} / \mathrm{kg}$ ) (B) and high-dose (10 mg/kg) (C) baicalin-treated groups $(\mathrm{P}<0.05)$. Data are expressed as mean \pm standard deviation and the number of lumens was counted at $40 x$ magnification. Scale bars: $50 \mu \mathrm{m}$.

supported by the observations of Deng et al. (10), who showed that baicalin is a potent inhibitor of renin and ACE. Previous studies showed that retinal angiogenesis is mediated by interaction between the angiotensin type 2 receptor, VEGF, and angiopoietin (11). In this study, the angiotensin type 2-receptor was expressed in the retina and was upregulated in oxygen-induced retinopathy, which suggests that retinal angiogenesis may be mediated by interactions between VEGF and angiotensin. Further studies are underway that are expected to reveal the role of baicalin in regulating these 2 pro-angiogenic molecules in the retina.

Our study shows that baicalin is a potent inhibitor of VEGF that can inhibit the formation of retinal neovascular tufts in vivo. It is generally thought that VEGF plays a crucial role in the process of neovascularization, and baicalin is known to inhibit VEGF, in addition to down-regulating angiotensin. Moreover, baicalin also inhibits the expression of MMP-2 and MMP-9 in the retina. These results suggest that the anti-angiogenic effect of baicalin occurs via multiple mechanisms, and further investigations are warranted to define the precise characteristics of this compound. Although studies have shown that baicalin can increase VEGF expression, our results clearly showed that this was not the case in ocular tissue. The reasons for this discrepancy are unclear; however, the study performed by Zhang et al. used fibroblasts and vascular endothelial cells, while our study was performed using an in vivo mouse model of OIR. Organ-specific responses to baicalin or differing environmental and developmental characteristics among in vitro and in vivo models may partially explain this discrepancy.

Traditionally, Scutellaria baicalensis has been used to treat various diseases, and this use provided the impetus to researchers to search for its active biological constituents, which ultimately led to the identification of 4 major flavonoids: wogonin, aglycone wogonin, baicalin, and baicalein. Although the anti-angiogenic effects of these compounds are still not clearly defined, their anti-oxidant capacities have been well documented $(12,13)$. Interestingly, baicalein is a more powerful antioxidant than baicalin, and thus further studies using baicalein in our experimental model should be considered. One of the great advantages of natural products for the treatment of human disease is that such products are easily ingestible for human subjects. The use of these compounds to inhibit pathologic angiogenesis is clearly worthy of consideration. Moreover, as pathologic angiogenesis is considered to be the main underlying pathophysiology in ocular diseases such as retinopathy of prematurity, age-related macular degeneration, and diabetic retinopathy, application of orally ingestible natural products such as baicalin in these diseases is of great promise.

In conclusion, our study provides evidence that a relative hyperbaric environment induces retinal angiogenesis by increasing angiotensin II and VEGF expression. Baicalin inhibited retinal angiogenesis by down-regulating pro-angiogenic proteins such as MMP-2, MMP-9, angiotensin II, and VEGF, and we suggest that this effect of baicalin plays an important role in its anti-angiogenic effect. Our data suggest that baicalin could benefit patients suffering from various ocular diseases, such as retinopathy of prematurity, age-related macular degeneration, and diabetic retinopathy, in which angiogenesis plays an important role. To validate this proposition, pharmacokinetic and pharmacodynamic studies should be performed to evaluate the bioavailability and mechanism of action of baicalin in vivo.

\section{MATERIALS AND METHODS}

\section{Animals}

Breeding pairs of ICR mice were originally purchased from Daehan Biolink (Seoul, Korea). All mice were provided food and water ad libitum and kept on a 12-hour light/dark cycle. Experiments were designed and performed in accordance with the guidelines of the ARVO Statement for the Use of Animals in Ophthalmic and Vision Research. Experimental protocols have been approved by the Institutional Animal Care and Use Committee of Incheon St. Mary's hospital (registration number: CIMC-2013-008).

\section{Oxygen-induced retinopathy in mice}

ICR pups were randomly divided into 4 groups: a normoxia 
group, an oxygen-exposed group (OIR group), and 2 groups that received different doses of baicalin. Each group had 1 nursing mother and 5-7 pups, and all experiments were repeated 3 times. At least 15 pups were included in each group. Oxygen-induced retinopathy was induced in ICR pups using previously published methods (14). For the OIR model, the newborn pups at post-natal day 7 (P7), along with their mothers, were transferred to a chamber supplied with $75 \pm 2 \%$ oxygen, which was under continual monitoring with a ProOx 110 oxygen controller (Biospherix, Ltd., Lacona, NY, USA) for $120 \mathrm{~h}$. On P12, the mice were returned to an atmosphere of normal room air and were given daily intraperitoneal (IP) injections of $10 \%$ DMSO in water (vehicle) or baicalin dissolved in vehicle at $1 \mathrm{mg} / \mathrm{kg}$ (B1, low-dose) or $10 \mathrm{mg} / \mathrm{kg}$ (B10, highdose). The mice in the normoxia group were maintained in normal room air from birth until P17.

\section{Whole flat mount fluorescent staining}

Retinal flat mount fluorescein staining was performed as previously described (15). Before injection, FITC-dextran (FD2000S; Sigma-Aldrich, St. Louis, MO, USA) was dissolved in ultrapure water at a concentration of $50 \mathrm{mg} / \mathrm{ml}$; this mixture was centrifuged at $10,000 \times g$ for 5 min and the supernatant was collected. FITC-dextran was protected from light during the preparation. In small animals such as pups, intravenous injection of FITC-dextran is technically challenging. Moreover, direct injection of the dye into the heart can directly damage it. Thus, retro-orbital injection of FITC-dextran was performed according to a previously published protocol (16). Mice were anesthetized on P17 by IP injection of ketamine (1\%), xylazine $(0.1 \%)$, and sodium chloride $(0.9 \%)$ at a dose of $0.1 \mathrm{ml} / 10 \mathrm{~g}$. A 27-gauge needle with a $1 \mathrm{ml}$ syringe was used to gently pierce 3-mm into the left orbital venous sinus with the bevel on the needle facing forward at a $45^{\circ}$ angle, and then $100 \mathrm{ml}$ of FITC-dextran was injected. Eyes were carefully harvested and fixed in $4 \%$ paraformaldehyde for $40 \mathrm{~min}$ at room temperature. The cornea, iris, lens, and vitreous humor were gently removed under a stereomicroscope (Leica, Wetzlar, Germany). Four to 6 radial incisions were made in the dissected retina, which was subsequently flattened with a coverslip. The vessels of the retina in each OIR mouse at P17 were viewed under a confocal microscope (Carl Zeiss, Jena, Germany). The retinal segments were merged to generate an image of the total retina (Photoshop CS3; Adobe Systems Inc., San Jose, CA, USA).

\section{Tissue section preparation and H\&E staining}

Pups from all groups were sacrificed on P17. Eyes of 4 mice from each group were analyzed (each experiment was performed at least 3 times, with 12 animals in each group). Eyes were removed, fixed in $4 \%$ paraformaldehyde in PBS for 24 hours, and embedded in paraffin. Sagittal sections of $8 \mu \mathrm{m}$ thickness, each $32 \mu \mathrm{m}$ apart, were cut parallel to the optic nerve. The sections were stained with hematoxylin and eosin (H\&E) to assess the vascular lumens of new vessels growing in- to the vitreous humor via light microscopy (Carl Zeiss, Chester, VA, USA). Vascular lumens between the lens and the inner limiting membrane were counted in at least 5 sections from each eye by 2 independent observers following a masked protocol. The mean number of neovascular lumens per section was calculated for each group.

\section{Quantification of retinal neovascularization}

Retinal neovascularization and vaso-obliteration were quantified as reported earlier (17). Both neovascularization and vasoobliteration are represented as \% of total retinal area. The total retinal area and vaso-obliteration quantifications were obtained using Adobe Photoshop CS3 as previously described (17). Neovascularization was quantified using a semi-automated computer program (SWIFT NV (18)), which is a set of macros run on NIH Imagej software. Artifacts like hyperfluorescent retinal ends, hyaloid vasculature, and cellular debris were excluded from the analysis. SWIFT NV divides the retinal image into 4 quadrants, and a threshold was set to highlight the neovascular tufts while excluding normal vasculature from the quantification. By this process, the program calculates the area of the neovascular tufts and generates a stitched image highlighting the neovascular tufts on the original retinal image.

\section{Westem blot analysis}

Western blot analysis was performed as previously described (19). For immunoblot analysis, the entire retina was lysed in RIPA buffer $(150 \mathrm{mM} \mathrm{NaCl}, 1.0 \%$ IGEPAL CA-630, 0.5\% sodium deoxycholate, $0.1 \%$ SDS, $50 \mathrm{mM}$ Tris, and $1 \mathrm{x}$ protease inhibitors; $\mathrm{pH}$ 8.0) and incubated for $30 \mathrm{~min}$ at $4{ }^{\circ} \mathrm{C}$. Samples were sonicated and centrifuged at $14,000 \times \mathrm{g}$ for $10 \mathrm{~min}$ at $4^{\circ} \mathrm{C}$, and the supernatant was transferred to a new microcentrifuge tube. The protein concentration in the entire retina was determined using a Bio-Rad Protein Assay kit (Bio-Rad Laboratories, Inc., Hercules, CA, USA). Next, an equal volume of $4 \mathrm{x}$ sample buffer (200 mM Tris, $8 \%$ SDS, $0.4 \%$ bromophenol blue, $40 \%$ glycine, and $400 \mathrm{mM}$ DTT; $\mathrm{pH}$ 6.8) was added to the sample, followed by boiling for $3-5 \mathrm{~min}$ at $100^{\circ} \mathrm{C}$. Samples were subsequently separated via $10-12 \%$ SDS-PAGE and transferred to a polyvinylidenedifluoride (PVDF) membrane (Hybond-P; GE Healthcare, Amersham, UK). After blocking with $5 \%$ skim milk in PBST buffer $(8 \mathrm{~g} / \mathrm{L} \mathrm{NaCl}, 0.2 \mathrm{~g} / \mathrm{L}$ $\mathrm{KCl}, 1.44 \mathrm{~g} / \mathrm{L} \mathrm{Na}_{2} \mathrm{HPO}_{4}, 0.24 \mathrm{~g} / \mathrm{L} \mathrm{NaH}_{2} \mathrm{HPO}_{4}$, and $0.1 \%$ Tween-20) for $30 \mathrm{~min}$, the membranes were incubated overnight at $4^{\circ} \mathrm{C}$ with the indicated primary antibody diluted in PBST containing $1 \%$ skim milk. Mouse monoclonal antibodies against VEGF $(1: 1,000)$ were purchased from Abcam (Cambridge, MA, USA). Rabbit polyclonal antibodies against angiotensin II $(1: 1,000)$ were purchased from Abcam (Cambridge, MA, USA). Rabbit monoclonal antibodies against MMP-2 $(1: 1,000)$, MMP-9 $(1: 1,000)$, and GAPDH $(1: 1,000)$ were purchased from Cell Signaling Technology (Beverly, MA, USA). Subsequently, the membranes were incubated with the appro- 
priate secondary antibody (horseradish peroxidase-conjugated goat anti-rabbit or horse anti-mouse secondary antibody at 1:3,000; Santa Cruz Biotechnology, Santa Cruz, CA, USA). The immunoreactive bands were visualized using enhanced chemiluminescence reagents (GE Healthcare, Amersham, UK) and then quantified via densitometry using an LAS-4000 image reader and Multi Gauge 3.1 software (Fuji Photo Film, Tokyo, Japan).

\section{Statistical analysis}

Each experiment was performed at least 3 times and the data were expressed as the mean \pm standard deviation (SD). The differences in central non-perfused area and neovascular tufts between the groups were compared using one way analysis of variance (ANOVA) followed by the Kruskal-Wallis test. The statistical analysis was performed using STATA/IC (version 11.2; StataCorp LP, College Station, TX, USA). P values less than 0.05 were considered statistically significant.

\section{REFERENCES}

1. Chow LC, Wright KW and Sola A (2001) Can changes in clinical practice decrease the incidence of severe retinopathy of prematurity in very low birth weight infants? Pediatrics 111, 339-345

2. Shalev B, Farr AK and Repka MX (2001) Randomized comparison of diode laser photocoagulation versus cryotherapy for threshold retinopathy of prematurity: seven-year outcome. Am J Ophthalmol 132, 76-80

3. Autrata R, Holousová M and Rehưrek J (2002) Cryotherapy and photocoagulation in the treatment of retinopathy of prematurity. Cesk Slov Oftalmol 58, 30-35

4. McDonald HR and Schatz H (1985) Visual loss following panretinal photocoagulation for proliferative diabetic retinopathy. Ophthalmology 92, 388-393

5. Lerner BC, Lakhanpal V and Schocket SS (1984) Transient myopia and accommodative paresis following retinal cryotherapy and panretinal photocoagulation. Am J Ophthalmol 97, 704-708

6. Liu JJ, Huang TS, Cheng WF and Lu FJ (2003) Baicalein and baicalin are potent inhibitors of angiogenesis: Inhibition of endothelial cell proliferation, migration and differentiation. Int J Cancer 106,559-565

7. Miocinovic R, McCabe NP, Keck RW, Jankun J, Hampton
JA and Selman SH (2005) In vivo and in vitro effect of baicalein on human prostate cancer cells. Int J Oncol 26, 241-246

8. Zhang K, Lu J, Mori T et al (2010) Baicalin increases VEGF expression and angiogenesis by activating the ERR $\{$ alpha\}/PGC-1 \{alpha\} pathway. Cardiovasc Res 89, 426-435

9. Yang SJ, Jo H, Kim JG and Jung SH (2014) Baicalin Attenuates Laser-Induced Choroidal Neovascularization. Curr Eye Res 39, 745-751

10. Deng YF, Aluko RE, Jin Q, Zhang Y and Yuan LJ (2012) Inhibitory activities of baicalin against renin and angiotensin-converting enzyme. Pharm Biol 50, 401-406

11. Sarlos S, Rizkalla B, Moravski CJ, Cao Z, Cooper ME and Wilkinson-Berka JL (2003) Retinal angiogenesis is mediated by an interaction between the angiotensin type 2 receptor, VEGF, and angiopoietin. Am J Pathol 163, 879887

12. Shieh DE, Liu LT and Lin CC (2000) Antioxidant and free radical scavenging effects of baicalein, baicalin and wogonin. Anticancer Res 20, 2861-2865

13. Huang WH, Lee AR and Yang $\mathrm{CH}$ (2006) Antioxidative and anti-inflammatory activities of polyhydroxyflavonoids of Scutellaria baicalensis GEORGI. Biosci Biotechnol Biochem 70, 2371-2380

14. Smith LE, Wesolowski E, McLellan A et al (1994) Oxygeninduced retinopathy in the mouse. Invest Ophthalmol Vis Sci 35, 101-111

15. Jo H, Jung SH, Kang J, Yim HB and Kang KD (2014) Sulodexide inhibits retinal neovascularization in a mouse model of oxygen-induced retinopathy. BMB Rep 47, 637-642

16. Nanni C, Pettinato C, Ambrosini V et al (2007) Retro-orbital injection is an effective route for radiopharmaceutical administration in mice during small-animal PET studies. Nucl Med Commun 28, 547-553

17. Connor KM, Krah NM, Dennison RJ et al (2009) Quantification of oxygen-induced retinopathy in the mouse: a model of vessel loss, vessel regrowth and pathological angiogenesis. Nat Protoc 4, 1565-1573

18. Stahl A, Connor KM, Sapieha P et al (2009) Computeraided quantification of retinal neovascularization. Angiogenesis 12, 297-301

19. Lim JH (2014) Zinc finger and BTB domain-containing protein 3 is essential for the growth of cancer cells. BMB Rep 47, 405-410 